\title{
Eficacia clínica de levofloxacina en el tratamiento de caninos con procesos infecciosos cutáneos y urinarios
}

\author{
Casas, L. ${ }^{1,2}$; Vaz, S. ${ }^{1}$; Landoni, M.F. ${ }^{1}$ \\ ${ }^{1}$ Cátedra de Farmacología General y Clínica; ${ }^{2}$ Hospital de Pequeños Animales, \\ Facultad Ciencias Veterinarias, UNLP, Calle 60 y 118, La Plata, Argentina. \\ E-mail: landoni@fcr.unlp.edu.ar
}

\begin{abstract}
Resumen
Casas, L.; Vaz, S.; Landoni, M.F.: Eficacia clínica de levofloxacina en el tratamiento de caninos con procesos infecciosos cutáneos y urinarios. Rev. Vet. 30: 1, 17-22, 2019. La eficacia y tolerancia de levofloxacina (Floxaday ${ }^{\circledR}$, Holliday-Scott) para el tratamiento de infecciones dérmicas y de vías urinarias, fue evaluada en caninos. Se incluyeron 26 casos clínicos que fueron tratados con $10 \mathrm{mg} / \mathrm{kg}$ vía oral cada 24 horas durante 14 y hasta 21 días. De los 14 casos de afecciones a nivel dérmico, 4 fueron severos y los restantes de severidad intermedia. De los 12 casos de infecciones a nivel urinario, 8 fueron procesos severos, 3 intermedios y uno bajo. La eficacia de los tratamientos fue evaluada aplicando una escala ad-hoc. El tratamiento fue exitoso en el $99 \%$ de los casos, observándose curación clínica al día 4 en el 6,25\% y al día 7 en el 43,75\%. En el día 14 la curación clínica fue registrada en el 99\% de los pacientes; solamente uno de ellos requirió un tratamiento de 21 días para alcanzarla. En la población con infecciones urinarias todos los pacientes respondieron satisfactoriamente al tratamiento (curación clínica), el $42 \%$ lo hizo hacia el día 4 y el $83 \%$ al día 7 . Al día 14 , la curación clínica fue constatada en el $100 \%$ de los casos. No se observaron efectos secundarios en ninguno de los pacientes incluidos en el estudio. En caninos, levofloxacina administrada por vía oral a la dosis de $10 \mathrm{mg} / \mathrm{kg}$ una vez al día, demostró una excelente eficacia y nula aparición de efectos adversos.
\end{abstract}

Palabras clave: canino, levofloxacina, eficacia, dermatitis, infección urinaria.

\begin{abstract}
Casas, L.; Vaz, S.; Landoni, M.F.: Clinical efficacy of levofloxacin on the treatment of dogs affected by skin and urinary infections. Rev. Vet. 30: 1, 17-22, 2019. The efficacy and tolerance of levofloxacin for the treatment of skin and urinary tract infections were evaluated in canines. Twenty six clinical cases were included and treated orally with $10 \mathrm{mg} / \mathrm{kg}$ every 24 hours for 14 to 21 days. From the 14 cases of skin disorders, 4 corresponded to severe cases and the rest to intermediate severity. From the 12 cases of infections at urinary level, 8 corresponded to severe infections, 3 were intermediate and one was low. Treatment was successful in $99 \%$ of the cases of dermatitis. Clinical cure (score 0 ) was observed at day 4 for $6.25 \%$ and day 7 for $43.75 \%$ of the animals, respectively. By day 14 clinical cure was observed in $99 \%$ of patients. One patient required a 21 -day treatment to reach score 0 . In the population with urinary infections, all patients responded satisfactorily to treatment (clinical cure-score 0 ), $42 \%$ on day 4 and $83 \%$ on day 7 . By day 14 clinical cure was observed in $100 \%$ of patients. No side effects were observed in any of the patients included in the study. Levofloxacin (Floxaday ${ }^{\circledR}$, Holliday-Scott) administered orally at a dose of $10 \mathrm{mg} / \mathrm{kg}$ once a day in canines, demonstrated excellent efficacy with no adverse effects.
\end{abstract}

Key words: canine, levofloxacin, efficacy, dermatitis, urinary tract infection.

\section{INTRODUCCIÓN}

Levofloxacina es un antimicrobiano perteneciente al grupo IIA de las fluoroquinolonas. Los miembros de este grupo han estado disponibles desde mediados de la década de 1980 y condujeron a importantes avances en la terapéutica de infecciones graves por bacterias

Recibido: 28 junio 2018 / Aceptado: 10 octubre 2018
Gram-negativas ${ }^{6,13}$. Estas drogas alcanzan elevadas concentraciones sistémicas cuando se administran por vía oral.

Levofloxacina es el isómero S(-) de ofloxacina. En este contexto, es importante remarcar que de los dos enantiómeros de ofloxacina $[\mathrm{R}(+)$ y $\mathrm{S}(-)]$, levofloxacina es el activo (eutómero) desde el punto de vista antimicrobiano (Landoni \& Albarellos, $J$ Vet Pharmacol Therap, en prensa, 2018). 
Las quinolonas son bactericidas de rápida acción, que actúan a través de la inhibición de las topoisomerasas bacterianas (enzimas que controlan el superenrollamiento y desenrollamiento del ADN bacteriano). La topoisomerasa afectada puede variar según el tipo de microorganismo: en gérmenes Gram negativos el sitio primario de unión es la topoisomerasa II o ADNgirasa, mientras que en estafilococos es la topoisomerasa IV. Estos contrastes en la afinidad por las enzimas no solo se reflejan en las diferencias en el espectro antibacteriano de cada fluoroquinolona, sino también en los mecanismos de resistencia bacteriana que pueden expresarse $^{7}$.

Como el resto de las fluoroquinolonas, levofloxacina está especialmente indicada para el tratamiento de infecciones urinarias, tejidos blandos, vías aéreas superiores e inferiores, piel, glándula mamaria, osteomielitis, peritonitis y septicemias producidas por gérmenes Gram negativos (E. coli y otras enterobacterias, Pseudomonas aeruginosa, Pasteurella sp y Bordetella bronchiseptica), también por algunos Gram positivos aerobios (estafilococos), infecciones por Mycoplasma y bacterias intracelulares, así como algunas micobacterias atípicas, estafilococos y Chlamydia/Chlamydophila $\mathrm{sp}^{12,14}$.

Desde el punto de vista farmacocinético, levofloxacina posee una excelente absorción oral, con relativamente baja unión a proteínas plasmáticas en la mayoría de las especies. Como el resto de las fluoroquinolonas, tiene una amplia distribución tisular, con volúmenes de distribución superiores al litro/kg. No sufre metabolización hepática y es excretada en forma activa fundamentalmente por orina ${ }^{3,7}$.

El objetivo del presente estudio fue evaluar la eficacia clínica de una nueva formulación de levofloxacina, aprobada por SENASA en septiembre del año 2017 (Floxaday ${ }^{\circledR}$, Laboratorios Holliday-Scott, Argentina), administrada por vía oral a la dosis de $10 \mathrm{mg} / \mathrm{kg} /$ día durante 14-21 días para el tratamiento de piodermias e infecciones del tracto urinario en caninos.

\section{MATERIAL Y MÉTODOS}

Se efectuó un estudio multi-céntrico, multi-investigador, paralelo, no randomizado y controlado, aplicándose un diseño del tipo "pre-test/post-test", siendo realizado por médicos veterinarios de la ciudad de Buenos Aires y del conurbano bonaerense.

Se incluyeron 26 casos clínicos, divididos en dos poblaciones, a saber: la población 1, que incluyó caninos de 1 a 10 años de edad $(n=14)$, con diagnóstico clínico de piodermia no asociada a enfermedades subyacentes endocrinas (hipotiroidismo, hiper-adrenocorticismo) y la población 2 , constituida por caninos de 1 a 10 años de edad, con diagnóstico clínico de infección en el aparato urinario $(n=12)$.

En ambas poblaciones se excluyeron los pacientes que presentaban una o más de las siguientes características: a) cachorros con menos de un año; b) caninos que hubieren recibido, dentro del periodo de 14 días previos, cualquier tipo de tratamiento antimicrobiano o antiinflamatorio (glucocorticoides, AINEs); c) caninos con enfermedades autoinmunes, neoplásicas o gastroentéricas; d) hembras preñadas o en lactancia; y e) caninos con parasitosis intestinal.

Para el análisis de la incidencia de efectos secundarios se consideró cada población siguiendo un diseño de grupo paralelo. En casos de no detectarse diferencias entre los grupos, se aplicaría un análisis descriptivo del pool de datos obtenidos.

Al momento de la primera consulta, en todos los casos (independientemente de la patología subyacente) se procedió a evaluar y registrar en la ficha clínica los datos anamnésicos, incluyendo: raza, peso, sexo, edad, alimento de consumo habitual, antecedentes de enfermedades y tratamientos recibidos previamente. Asimismo se realizó una evaluación clínica general (actitud, apetito y dolor) y de acuerdo al cuadro patológico, se continuó con la evaluación particular.

Las evaluaciones particulares se repitieron los días 4, 7 y 14 post-tratamiento. Los estudios complementarios propios de cada cuadro patológico se realizaron el día 1 y se repitieron el último día de tratamiento. En caso de que el responsable médico lo creyera necesario era posible la realización de estudios complementarios en fechas adicionales.

La eficacia clínica de levofloxacina administrada por vía oral a la dosis de $10 \mathrm{mg} / \mathrm{kg} / 24 \mathrm{~h}$ fue evaluada a través de la evolución de distintos parámetros clínicos. Estos parámetros fueron divididos en generales y específicos de las patologías incluidas en el estudio.

A efectos de aplicar tests estadísticos, se procedió a graduar los parámetros clínicos incluidos en la evaluación, tanto los generales como los específicos. Para ello, se crearon escalas ad-hoc que permitieron generar scores para cada uno de los parámetros. Las escalas ad-hoc para cuantificar la severidad de las lesiones a través de los parámetros clínicos generales así como de los específicos para cada población en estudio, se presentan en la Tabla 1.

Población 1. Como se mencionara previamente, esta población estuvo conformada por 14 casos. Del total de animales, 10 correspondieron a pacientes machos y los restantes 4, a hembras enteras. El peso promedio de la población estudiada fue de $21,75 \mathrm{~kg}$ con un desvío estándar de $11,93 \mathrm{~kg}$ y una mediana de $25 \mathrm{~kg}$, mientras que la edad promedio fue de 3,96 años con un desvío estándar de 3,69 y una mediana de 3,5 años.

La población incluyó las siguientes razas: schnauzer mini, sharpei, caniche, dobermann, boxer, ovejero alemán, golden retriever y mestizos.

Los diagnósticos definitivos y la severidad de los casos incluidos en la población (severidad calificada en el rango de 1 a 3 , equivaliendo 3 a la calificación más alta), fueron: un caso de foliculitis superficial (severidad 2); un caso de alopecia de color diluido (severidad 3 ); un caso de furunculosis (severidad 3); seis casos de 
demodicosis con foliculitis (severidad 2); un caso de demodicosis con piodermia profunda (severidad 2$)$; un caso de dermatitis alérgica con piodermia (severidad 2); un caso de herida traumática de evolución crónica con miasis (severidad 3); un caso de piodermia (severidad 2); y un caso de piodermia perianal con fístula (severidad 3).

Población 2. Del total de 12 casos, 10 correspondieron a pacientes machos y los restantes a una hembra entera y otra castrada. El peso promedio de la población estudiada fue de $26,43 \mathrm{~kg}$ con un desvío estándar de $22,19 \mathrm{~kg}$ y una mediana de $26 \mathrm{~kg}$, mientras que la edad promedio fue de 7,65 años con un desvío estándar de 3,15 y una mediana de 7,5 años.

En la población se incluyeron las siguientes razas: ovejero belga, pekinés, caniche, lebrel irlandés, dachshund, ovejero alemán, pastor suizo y mestizos. Los diagnósticos definitivos y la severidad de los casos incluidos en la población (calificada en el rango de 1 a 3, equivaliendo 3 a la calificación más alta), fueron: dos casos de infección del tracto urinario (severidad 2); cuatro casos similares al anterior (severidad 3); un caso de prostatitis (severidad 3); dos casos de cistitis (severidad 1) y tres casos de pielonefritis (severidad 2).

Los datos recogidos fueron analizados estadísticamente, en primer lugar de manera descriptiva, calculando medidas de tendencia central y dispersión (media aritmética y desvío estándar). Posteriormente, las diferencias entre las puntuaciones a tiempo 0 y los distintos tiempos post-tratamiento, con un límite de significación de $0,05(\mathrm{p}<0,05)$, fueron evaluadas aplicando el test de Mann Withney. Todos los análisis estadísticos fueron realizados con el programa GraphPad Prism ${ }^{\circledR}$.

\section{RESULTADOS}

Por fallas o incumplimiento del protocolo, seis casos debieron retirarse del estudio; cuatro de ellos correspondieron a la población 2 y los restantes a la población 1. Las causas se detallan a continuación:

-Caso 1. No se incluyó la evaluación del día 0 .

-Casos 2, 3 y 4 . Tienen score 0 al día 0 ; no está justificada la indicación del tratamiento.

-Caso 5. No figuran los seguimientos.

-Caso 6. No se pudo confirmar la correcta administración de la dosis por dificultades en la aceptación de la medicación por el paciente.

Población 1. De los 14 casos estudiados (Tabla 1 y Figura 1) todos, con excepción del caso 14, finalizaron el tratamiento con la curación clínica de la patología infecciosa blanco $(\mathrm{p}<0,05)$.

En ninguno de los casos clínicos estudiados se observó algún tipo de efecto adverso. Cabe aclarar que el paciente 2, que poseía antecedentes de efectos desfavorables a nivel gastrointestinal con otros tratamientos antibióticos orales, no mostró ningún tipo de efecto adverso a ese nivel.
Tabla 1. Escalas ad-hoc para cuantificar la severidad de los parámetros clínicos de las diferentes patologías evaluadas.

\begin{tabular}{ll}
\hline Parámetros clínicos generales \\
& 0 - normal \\
actitud & 1 - levemente deteriorada \\
en reposo & 2 - severamente deteriorada \\
& 3 - postrado \\
\hline \multirow{2}{*}{ apetito } & 0 - normal \\
& 1 - leve disminución del consumo \\
& 2 - severa disminución del consumo \\
& 3 - anorexia \\
\hline \multirow{2}{*}{ dolor } & 0 - ausente \\
& 1 - leve \\
& 2 - moderado \\
& 3 - severo \\
\hline
\end{tabular}

\section{Parámetros clínicos específicos de la población 1}

\begin{tabular}{ll} 
& 0 - ausente \\
prurito & 1 - leve \\
& 2 - moderado \\
& 3 - severo \\
\hline & 1 - pápulas \\
& 2 - pústulas \\
presencia & 3 - collaretes \\
de lesiones & 4 - forúnculos \\
& 5 - fístulas \\
& 6 - ulceras \\
\hline & $1 \leq 10$ \\
porcentaje corporal & $2 \leq 25$ \\
con presencia & $3 \leq 50$ \\
de lesiones & $4 \leq 75$ \\
& $5>75 \%$ \\
\hline
\end{tabular}

Parámetros clínicos específicos de la población 2

\begin{tabular}{ll} 
diuresis & 0 - normal \\
& 1 - poliuria \\
& 2 - polaquiuria/hematuria \\
& 3 - anuria \\
\hline úlceras en & 0 - ausentes \\
cavidad bucal & 1 - presentes \\
\hline aliento amoniacal & 0 - ausente \\
& 1 - presente \\
\hline descarga prepucial/ & 0 - ausente \\
vaginal (ponderando & 2 - serosa \\
fase de ciclo estral) & 3 - purulenta abundante \\
\hline
\end{tabular}

En el caso 14, una paciente hembra de 8 años con demodicosis complicada con piodermia, el médico veterinario responsable decidió la finalización del tratamiento el día 14. Si bien la paciente había mostrado mejoría inicial (el día 7 se observó una caída del puntaje de 7 unidades $(p<0,05)$, al considerar que el día 14 no se había profundizado la mejoría, el profesional decidió finalizar el tratamiento e iniciar una interconsulta con un endocrinólogo debido a la sospecha de la presencia de hipotiroidismo.

De los pacientes que respondieron satisfactoriamente al tratamiento (curación clínica, puntuación 0), el 6,25\% lo hizo el día 4 y el $43,75 \%$ el día 7 . Para el día 14 la curación clínica fue observada en el $99 \%$ de los pacientes. Solo un paciente (caso 5) requirió un tratamiento de 21 días para alcanzar el score 0 , o sea curación clínica. 
Tabla 2. Puntuaciones en los distintos tiempos de evaluación de los pacientes con infecciones de piel.

\begin{tabular}{lccccc}
\hline \multirow{2}{*}{ caso } & \multicolumn{5}{c}{ día de tratamiento } \\
\cline { 2 - 6 } & 0 & 4 & 7 & 14 & 21 \\
\hline 1 & 10 & 9 & 6 & 0 & NA \\
2 & 5 & 5 & 2 & 0 & $"$ \\
3 & 9 & 8 & 5 & 0 & $"$ \\
4 & 5 & 5 & 3 & 0 & $"$ \\
5 & 9 & 4 & 4 & 3 & 0 \\
6 & 9 & 5 & 2 & 0 & NA \\
7 & 8 & 5 & 4 & 0 & $"$ \\
8 & 5 & 5 & 0 & 0 & $"$ \\
9 & 5 & 3 & 0 & 0 & $"$ \\
10 & 5 & 5 & 0 & 0 & $"$ \\
11 & 3 & 0 & 0 & 0 & $"$ \\
12 & 6 & 5 & 0 & 0 & $"$ \\
13 & 14 & 14 & 12 & 0 & $"$ \\
14 & 13 & 11 & 6 & 6 & $"$ \\
\hline $\mathrm{X}$ & 7,57 & 6,00 & 3,14 & 0,64 & $"$ \\
EE & 0,88 & 0,93 & 0,91 & 0,46 &, \\
\hline
\end{tabular}

X: media aritmética, EE: error estándar, NA: no aplicable.

Población 2. De los 12 casos estudiados (Tabla 3 y Figura 2), todos finalizaron el tratamiento con curación clínica de la patología infecciosa blanco (puntuación final 0). No se reportaron efectos adversos en ninguno de los casos clínicos estudiados.

De los pacientes que respondieron satisfactoriamente al tratamiento (curación clínica, puntuación 0), el $42 \%$ lo hizo el día 4 y el $83 \%$ el día 7 . Para el día 14 la curación clínica fue observada en el $100 \%$ de los pacientes.

\section{DISCUSIÓN}

En el presente estudio levofloxacina demostró una alta eficacia, tanto en patologías a nivel cutáneo como urinario. De la totalidad de casos estudiados solo uno
Tabla 3. Puntuaciones en los distintos tiempos de evaluación de los pacientes con infecciones urinarias.

\begin{tabular}{|c|c|c|c|c|}
\hline \multirow{2}{*}{ caso } & \multicolumn{4}{|c|}{ día de tratamiento } \\
\hline & 0 & 4 & 7 & 14 \\
\hline 1 & 2 & 2 & 0 & 0 \\
\hline 2 & 2 & 2 & 0 & $"$ \\
\hline 3 & 3 & 0 & 0 & $"$ \\
\hline 4 & 2 & 2 & 2 & $"$ \\
\hline 5 & 5 & 3 & 0 & $"$ \\
\hline 6 & 5 & 2 & 0 & $"$ \\
\hline 7 & 3 & 3 & 1 & $"$ \\
\hline 8 & 5 & 2 & 0 & $"$ \\
\hline 9 & 5 & 0 & 0 & $"$ \\
\hline 10 & 1 & 0 & 0 & NA \\
\hline 11 & 1 & 0 & 0 & ", \\
\hline 12 & 2 & 0 & 0 & $"$ \\
\hline $\mathrm{X}$ & 3,00 & 1,33 & 0,25 & $"$ \\
\hline $\mathrm{EE}$ & 0,46 & 0,35 & 0,18 & $"$ \\
\hline
\end{tabular}

X: media aritmética, EE: error estándar, NA: no aplicable.

requirió un tratamiento de 21 días. Los restantes mostraron curación clínica (puntuación 0) al día 14 de tratamiento.

Levofloxacina, al igual que marbofloxacina, orbifloxacina y pradofloxacina, posee una sustitución a nivel del carbono-8. Estas moléculas son denominadas fluoroquinolonas de tercera generación para diferenciarlas de las de segunda generación, como ciprofloxacina y enrofloxacina ${ }^{4}$.

Las fluoroquinolonas de tercera generación se caracterizan por una muy alta eficacia frente a bacterias Gram (-), manteniendo una actividad frente a bacterias Gram (+) similar a las de segunda generación ${ }^{4}$. Los presentes resultados reflejan la alta eficacia clínica de las fluoroquinolonas de tercera generación.

La eficacia de levofloxacina en infecciones con asiento en piel, como la observada en el presente estudio, es comparable con la reportada por algunos

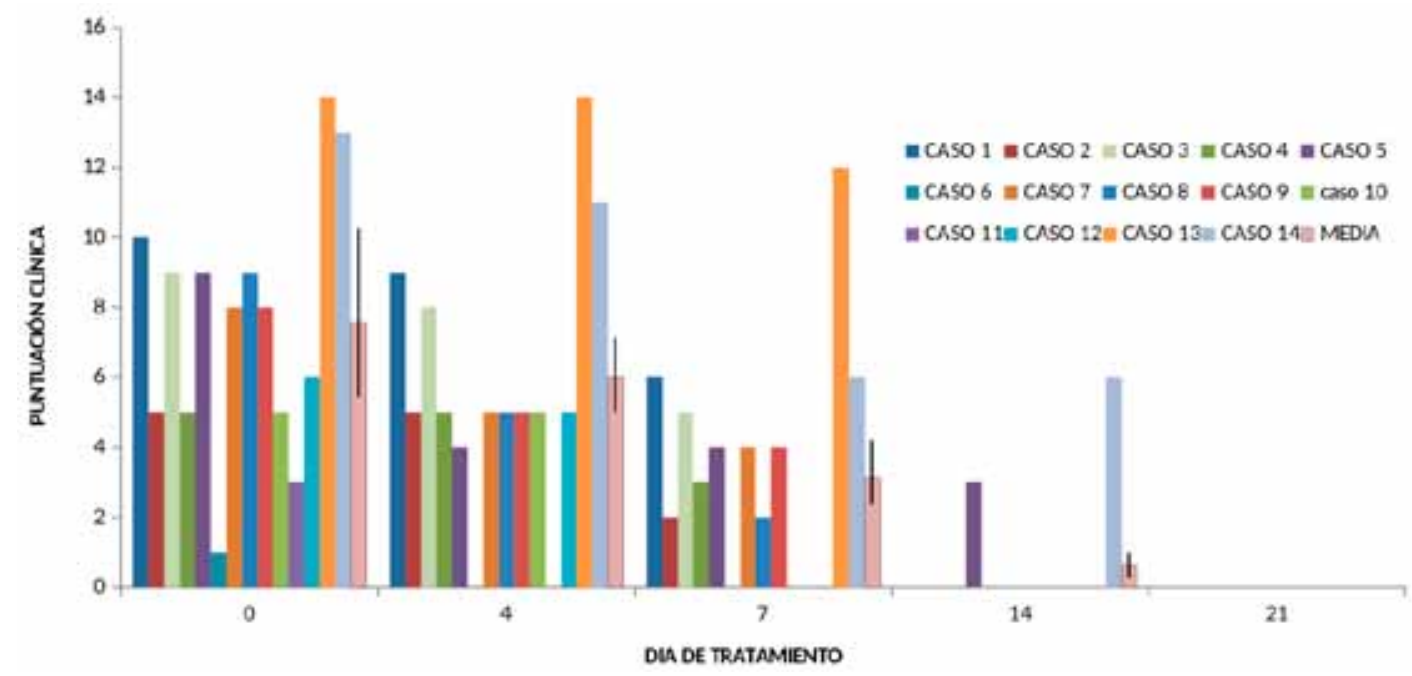

Figura 1. Evolución de scores clínicos $(\mathrm{X} \pm \mathrm{EE})$ en los distintos tiempos de evaluación en pacientes con infecciones de piel. 


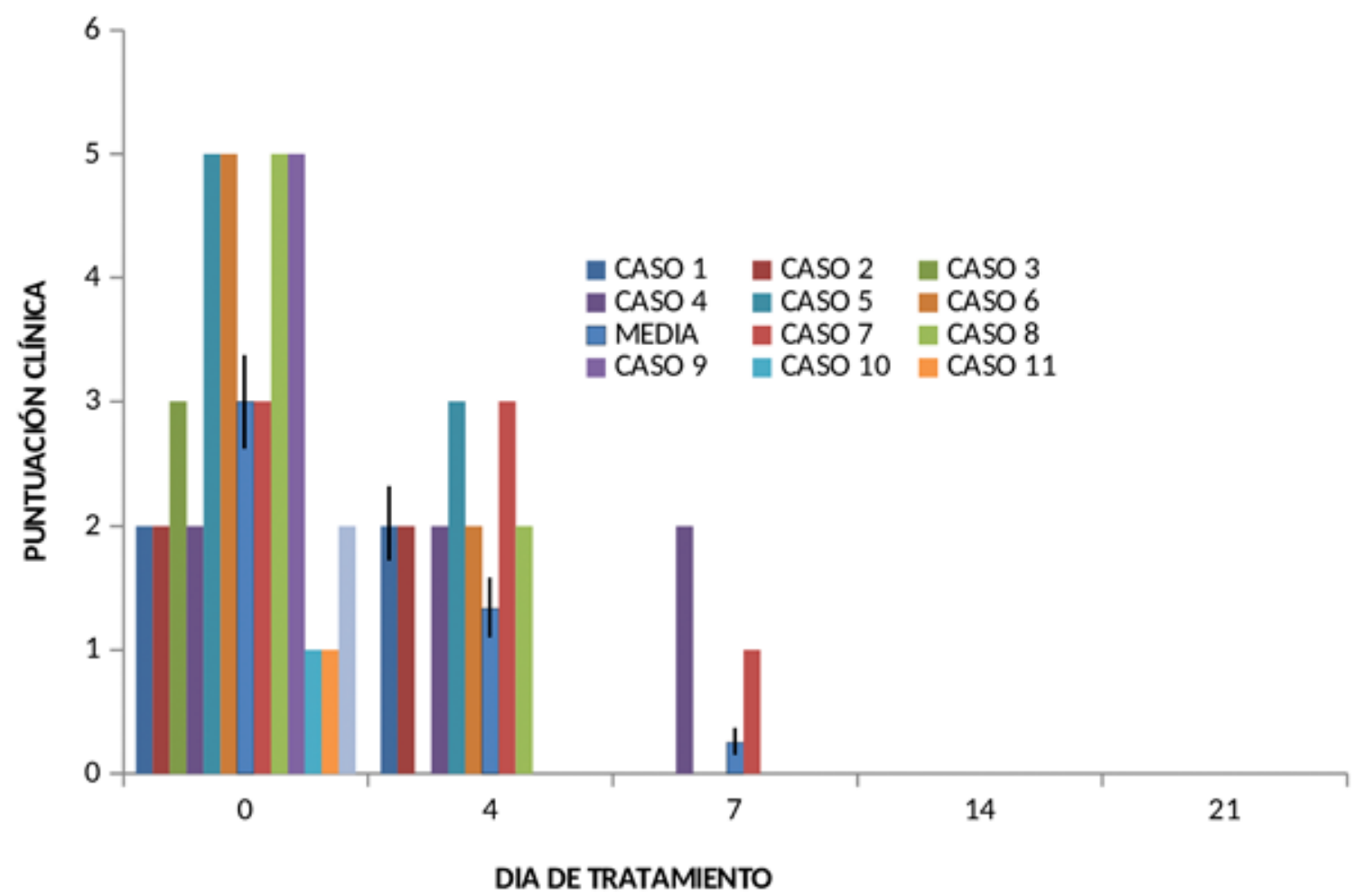

Figura 2. Evolución de scores clínicos $(\mathrm{X} \pm \mathrm{EE})$ en los distintos tiempos de evaluación en pacientes con infecciones urinarias.

investigadores para pradofloxacina $9,10,11$ y para marbofloxacina por otros ${ }^{12}$. Sin embargo, tanto para pradofloxacina como para marbofloxacina se requirieron tratamientos más prolongados (entre 21 y 28 días).

Considerando que las fluoroquinolonas tienen una cinética de muerte bacteriana del tipo concentración dependiente ${ }^{8}$, esta diferencia podría deberse a la menor concentración máxima observada (Cmax) reporta$\mathrm{da}$ en caninos tanto para pradofloxacina $(1,2 \mu \mathrm{g} / \mathrm{ml})^{2,5}$ como para marbofloxacina $(1,35 \mu \mathrm{g} / \mathrm{ml})^{1}$, en comparación con la reportada para levofloxacina $(3,20 \mu \mathrm{g} / \mathrm{ml})$.

Las fluoroquinolonas en general, y las de tercera generación en particular, poseen una alta eficacia para el tratamiento de patologías a nivel urinario ${ }^{3}$. En el presente estudio, levofloxacina demostró una tasa de curación clínica al día 7 del $83 \%$, alcanzando el 100\% al día 14. Estos resultados reflejan la potencia de levofloxacina frente a los patógenos más comúnmente asociados a las infecciones del tracto urinario en caninos.

El rango de valores de las concentraciones inhibitorias mínimas (CIM) para levofloxacina frente a cepas de $E$. coli uropatogénicas multiresistentes con sensibilidad a enrofloxacina aisladas de caninos, ha sido reportada entre 0,004 y $0,25 \mu \mathrm{g} / \mathrm{ml}^{6}$. Considerando el valor mayor de CIM, la Cmax de levofloxacina tras su administración oral representaría casi 13 veces este valor; considerando que, como se mencionó previamente, levofloxacina pertenece al grupo de antimicrobianos concentración dependiente, dato que explica su elevada potencia.

En conclusión, los resultados del presente estudio sugieren que Floxaday ${ }^{\circledR}$ es una nueva y eficaz alterna- tiva para el tratamiento sistémico de infecciones con asiento en piel y tracto urinario. La ausencia de efectos adversos y su conveniente régimen de administración (una vez al día por vía oral), la transforman en una valiosa alternativa terapéutica en caninos.

Agradecimiento. A la firma Holliday-Scott, Argentina, por el apoyo económico.

\section{REFERENCIAS}

1. Cester CC, Schneider M, Toutain PL. 1996. Comparative kinetics of two orally administered fluoroquinolones in dog: enrofloxacin versus marbofloxacin. Rev Med Vet 47: 703-716.

2. Fraatz K, Krebber R, Edingloh M, Heinen E. 2003. Oral bioavailability of pradofloxacin tablets and renal excretion in dogs. J Vet Pharm Therap 26: 88-89.

3. Howes C, Tappin S. 2016. Canine urinary tract infections. Companion Anim 21: 100-108.

4. King DE, Malone R, Lilley SH. 2000. New classification and update on the quinolone antibiotics. Am Fam Physician 61: 2741-2748.

5. Lees P. 2013. Pharmacokinetics, pharmacodynamics and therapeutics of pradofloxacin in the dog and cat. $J$ Vet Pharmacol Therap 36: 209-221.

6. Liu X, Boothe D, Jin Y, Thungrat K. 2013. In vitro potency and efficacy favor later generation fluoroquinolones for treatment of canine and feline Escherichia coli uropathogens in the United States. World J Microbiol Biot 29: 347-354. 
7. Martinez M, McDermott P, Walker R. 2006. Pharmacology of the fluoroquinolones: a perspective for the use in domestic animals. Vet J. 172: 10-28.

8. McKellar QA, Sanchez SF, Jones DG. 2004. Pharmacokinetic / pharmacodynamic relationships of antimicrobial drugs used in veterinary medicine. $J$ Vet Pharmacol Therap 27: 503-514.

9. Mueller RS, Stephan B. 2007. Pradofloxacin in the treatment of canine deep pyoderma: a multicentred, blinded, randomized parallel trial. Vet Dermatol 18: 144-151.

10. Paradis $\mathbf{M}$ et al. 2001. Evaluation of the clinical efficacy of marbofloxacin (Zeniquin) tablets for the treatment of canine pyoderma: an open clinical trial. Vet Dermatol 12: 163-169.
11. Restrepo C, Ihrke PJ, White SD, Spiegel IB, Affolter VK. 2010. Evaluation of the clinical efficacy of pradofloxacin tablets for the treatment of canine pyoderma. $\mathrm{J} \mathrm{Am}$ Anim Hosp Assoc 46: 301-311.

12. Riddle C, Lemons CL, Papich MG, Altier C. 2000. Evaluation of ciprofloxacin as a representative of veterinary fluoroquinolones in susceptibility testing. $J$ Clin Microbiol 38: 1636-1637.

13. Toutain PL, Castillo JR, Bousquet A. 2002. The pharmacokinetic-pharmacodynamic approach to a rational dosage regimen for antibiotics. Res Vet Sci 73: 105-114.

14. Walker RD. 2000. The use of fluoroquinolones for companion animal antimicrobial therapy. Australian Vet J 78: 84-90.

\section{Revista Veterinaria ingresa a SciELO}

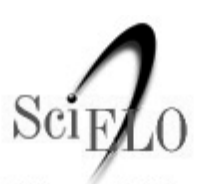

Scientific Electronic Library Online

Revista Veterinaria, publicación oficial de la Facultad de Ciencias Veterinarias de la Universidad Nacional del Nordeste (Corrientes, Argentina), ha logrado acceder al Núcleo Básico de Revistas Científicas Argentinas (Nivel 1), luego de calificar adecuadamente en el Centro Argentino de Información Científica y Tecnológica (CAICYT), según Resolución 2485/14 del Consejo Nacional de Investigaciones Científicas y Técnicas (CONICET).

Sobre un puntaje máximo de 33 se obtuvieron 32 puntos. Tal calificación constituye "una garantía de la excelencia de la publicación" (sic) y queda expedita la vía del Portal SciELO (Scientific Electronic Library Online) para los artículos publicados.

En tal calificación gravitó positivamente la circunstancia de haber aumentado el índice de impacto (Scimago-Elsevier) y haber disminuido las autocitaciones. También se tuvieron en cuenta aspectos como la amplia cobertura de la revista, la calidad científica del Comité Editorial, los criterios de evaluación de los artículos, el origen de los autores (locales $60 \%$, nacionales $13 \%$, extranjeros $27 \%$, en idioma inglés), el adecuado balance entre trabajos científicos originales y reseñas bibliográficas (ambos con alta calidad), así como el estricto cumplimiento de la periodicidad semestral y la favorable acogida por indizadores como Cab, J-Gate, Doaj, Ebsco, Gale Cengage, Infocyt, Latindex y Scopus.

Se consolida de esta manera la continuidad de "Revista Veterinaria", que en su acontecer registra más de 50 años de existencia en nuestra Facultad de Ciencias Veterinarias, entidad que en 2018 cumplió el $98^{\circ}$ aniversario de su fundación. 